\title{
Uppsala University
}

This is a submitted version of a paper published in Nuclear Instruments and Methods in Physics Research Section A: Accelerators, Spectrometers, Detectors and Associated Equipment.

Citation for the published paper:

Andersson Sundén, E., Ballabio, L., Cecconello, M., Conroy, S., Ericsson, G. et al. (2013)

"Evaluation of neutron spectrometer techniques for ITER using synthetic data" Nuclear Instruments and Methods in Physics Research Section A: Accelerators, Spectrometers, Detectors and Associated Equipment, 701: 62-71

URL: http://dx.doi.org/10.1016/j.nima.2012.10.041

Access to the published version may require subscription.

Permanent link to this version:

http://urn.kb.se/resolve?urn=urn:nbn:se:uu:diva-121519

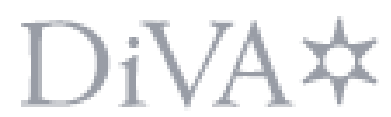

http://uu.diva-portal.org 


\title{
Evaluation of neutron spectrometer techniques for ITER using synthetic data
}

E.Andersson Sundén, L. Ballabio ${ }^{1}$, M.Cecconello, S.Conroy, G.Ericsson, M.Gatu Johnson, G.Gorini ${ }^{1}$, C.Hellesen, F.Ognissanto ${ }^{1}$, E.Ronchi, H.Sjöstrand, M.Tardocchi ${ }^{1}$ and M.Weiszflog.

Department of Physics and Astronomy, EURATOM-VR Association, Uppsala, Sweden

${ }^{1}$ Istituto di Fisica del Plasma, EURATOM-ENEA-CNR Association, and CNR-CNISM, Milan, Italy

\begin{abstract}
A neutron spectrometer at ITER is expected to provide estimates of plasma parameters such as ion temperature, $T_{\mathrm{i}}$, fuel ion ratio, $n_{\mathrm{t}} / n_{\mathrm{d}}$, and $Q_{\text {thermal }} / Q_{\text {tot }}$, with $10-20 \%$ precision at a time resolution, $\Delta t$, of at least $100 \mathrm{~ms}$. The present paper describes a method for evaluating different neutron spectroscopy techniques based on their instrumental response functions and synthetic measurement data. We include five different neutron spectrometric techniques with realistic response functions, based on simulations and measurements where available. The techniques are magnetic proton recoil, thin-foil proton recoil, gamma discriminating organic scintillator, diamond and time-of-flight. The reference position and line of sight of a high resolution neutron spectrometer on ITER are used in the study. ITER plasma conditions are simulated for realistic operating scenarios. The ITER conditions evaluated are beam and radio frequency heated and thermal deuterium-tritium plasmas. Results are given for each technique in terms of the estimated time resolution at which the parameter determination can be made within the required precision (here $10 \%$ for $T_{\mathrm{i}}$ and the relative intensities of NB and RF emission components). It is shown that under the assumptions made, the thin-foil techniques out-perform the other spectroscopy techniques in practically all measurement situations. For thermal conditions, the range of achieved $\Delta t$ in the determination of $T_{\mathrm{i}}$ varies in time scales from ms (for the magnetic and thin-foil proton recoil) to s (for gamma discriminating organic scintillator).
\end{abstract}




\section{Introduction}

Neutrons are emitted from a deuterium (D) and tritium (T) plasma at energies around $2.5 \mathrm{MeV}$ and $14 \mathrm{MeV}$ from the $\mathrm{d}+\mathrm{d} \rightarrow{ }^{3} \mathrm{He}+\mathrm{n}$ and $\mathrm{d}+\mathrm{t} \rightarrow \alpha+\mathrm{n}$ reactions, respectively. Neutrons leave the magnetic confinement of a tokamak unhindered and carry information from the core of the plasma. The energy distribution of the neutron emission is intimately coupled to the plasma's ion velocity distributions. Plasma parameters such as fusion power, $P_{\text {fusion }}[1]$, ion temperature, $T_{\mathrm{i}}$ [2], thermal fraction of total $Q, Q_{\mathrm{thermal}} / Q_{\mathrm{tot}}$ [3], the fuel ion ratio, $n_{\mathrm{t}} / n_{\mathrm{d}}$ [4], and the plasma rotation [2] as well as the presence of fast ions [5] and impurities [6] can be measured with neutron spectrometry.

Presently, a high resolution neutron spectrometer (HRNS) on ITER falls in the "enabled" category, meaning that only the basic interfacing for the instrument will be provided. In the case of HRNS, a reference position and sight line are allocated in equatorial port cell \#1 but further instrumental details and funding are pending. The reference line of sight (LOS) is close to radial (at a $98^{\circ}$ inclination angle to the B-field in the centre of the plasma) and has a so far undetermined diameter of the first wall aperture in the range 100-300 mm. Interfacing issues are important, in particular for HRNS techniques that are limited by the available neutron flux. The flux scales linearly with the first wall aperture area, the fusion power and the active area of the spectrometer.

The present paper evaluates the performance of five spectrometric techniques, measuring the emission from the $\mathrm{d}+\mathrm{t}$ reaction, in terms of the time resolution at which they are able to fulfil the ITER requirements in determining $T_{\mathrm{i}}$ and $Q_{\text {thermal }} / Q_{\text {tot }}$ at $10 \%$ precision; the required time resolution is $100 \mathrm{~ms}$ [7]. We have assumed the reference HRNS position and LOS and used the smaller first wall diameter $(100 \mathrm{~mm})$. The evaluation has been performed using synthetic measurement data to assess the accuracy and precision of the estimated parameter value, using the same method as presented in $[8,9,10,11]$.

The paper is organized in the following way: Section 2 describes the evaluation method. The five measurement techniques are reviewed and their instrument response functions (IRFs) are given in Section 3. In Section 4, the ITER plasma conditions are presented in terms of thermal as well as neutral beam injection (NBI) and ion cyclotron resonance heating (ICRH) ion populations. The neutron emission components associated with these ion populations are also described. The results of the evaluation are given in Section 5, which is followed by a discussion and conclusions in Sections 6 and 7, respectively.

\section{Evaluation method}

To assess the time resolution that can be achieved by different HRNS techniques in determining a number of pertinent ITER plasma parameters (here mainly the ion temperature and the contributions of the non-thermal emission components to the total Q), modelled neutron spectra are used in combination with best-estimate IRFs. The first step in the method is to produce the 


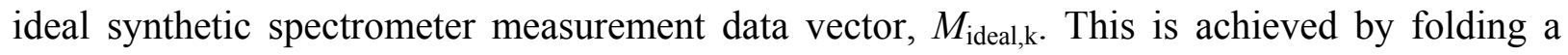
modelled neutron emission spectrum, $I\left(E_{\mathrm{n}}\right)$, with the IRF of the selected HRNS technique:

$$
M_{\text {ideal }, \mathrm{k}}=\sum_{\mathrm{i}}\left(I R F_{\mathrm{i}, \mathrm{k}} \cdot I_{\mathrm{i}}\right)
$$

Here, $I R F$ is the discrete instrument response function (in units of efficiency per incoming neutron), in the form of a matrix with indices $i$ for neutron energy bin and $k$ for the instrument's physical measurements parameter (detector channel, $t_{\mathrm{TOF}}$ bin, pulse height bin, etc.). $I$ is the (discrete) modelled neutron emission energy spectrum. In general, the ITER neutron energy spectrum is constructed from pre-calculated spectral components (models) corresponding to fusion reactions involving thermal, beam-thermal and ICRH-thermal fuel ion populations (see Sect. 4). In Figure $1 \mathrm{a}$ and $1 \mathrm{~b}$, a modelled neutron spectrum from a plasma in thermal equilibrium and $M_{\text {ideal }}$ and $M_{\text {meas }}$ (for a technique with a box-shaped IRF) are shown, respectively. A synthetic measurement vector, $M_{\text {meas }}$, is obtained by imposing a random Poisson fluctuation to each bin of $M_{\text {ideal }}$.

To obtain an estimate of the sought-after plasma parameters the process is run in reverse, using the synthetic $M_{\text {meas }}$ in a fitting procedure using a standard minimising technique and with the original parameters of the plasma components as free parameters. Here we chose to minimise the metric $C$ as suggested in [12],

$$
C=-2 \sum_{\mathrm{j}}\left\{M_{\text {meas }, \mathrm{j}} \cdot \ln \left(N_{\mathrm{j}}\right)-N_{\mathrm{j}}-\ln \left(M_{\text {meas } \mathrm{j}} \mathrm{j}\right)\right\},
$$

where $M_{\text {meas,j }}$ is the synthetic measurement data and $N_{\mathrm{j}}$ is the folded model data in bin $j$. Note that $C$ is the metric corresponding to Poisson distributed data, in the same way as $\chi^{2}$ is associated with Gaussian statistics.
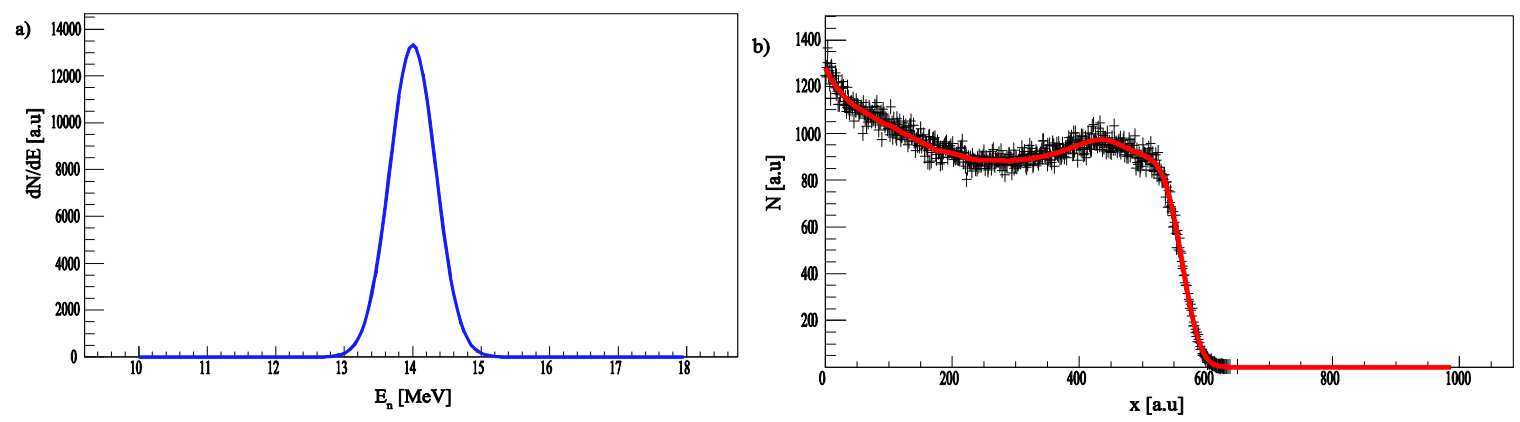

Figure 1. (a) Modelled neutron spectrum from a plasma in thermal equilibrium. (b) Ideal synthetic data, $M_{\text {ideal }}$ (red), together with synthetic measurement data, $M_{\text {meas }}$ (crosses), of a HRNS technique with a box-like IRF.

The evaluation procedure is repeated 100 times for each given scenario, to give a reasonable set of estimated parameter values on which a statistical analysis can be performed. The precision of the parameter estimate is given by the standard deviation, $\sigma$, of the resulting parameter distribution obtained in the evaluation procedure, and the accuracy is given by the average offset of the parameters from the true value (which is exactly known). In Figure 2, the distribution of $T_{\mathrm{i}}$ obtained from the evaluation is shown for a technique with a box-shaped IRF. 
The accuracy is within the expected standard deviation, $\sigma$, of the mean, $\sigma / n^{0.5}$, where $n$ is the number of method cycles. An overview of the evaluation procedure is illustrated in Figure 3.

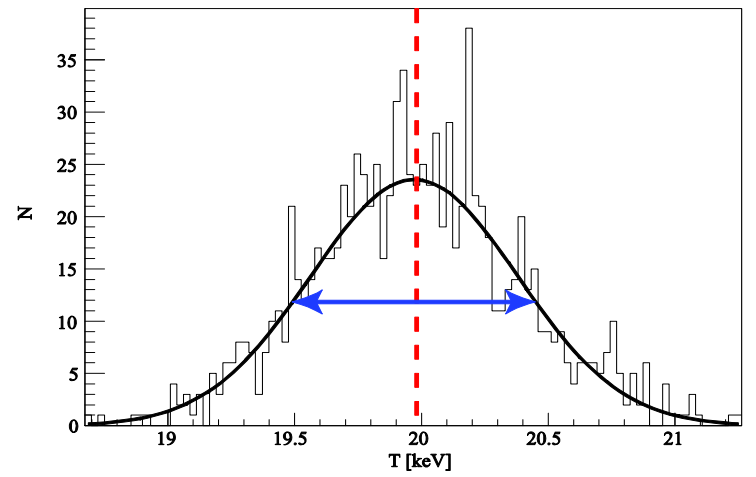

Figure 2. Temperature distribution of 1000 evaluation cycles for a technique with box-shaped IRF $\left(T_{i}=20 \mathrm{keV}\right)$ together with a Gaussian fit to the distribution. Indicated are also the precision (blue arrow) and the mean value of the distribution (red dashed line). The accuracy is the deviation of the mean value of the distribution from the true value. Here the precision $\left(\sigma / E_{n}\right)$ is $2.1 \%$ and the accuracy is $5 \cdot 10^{-2} \%$.

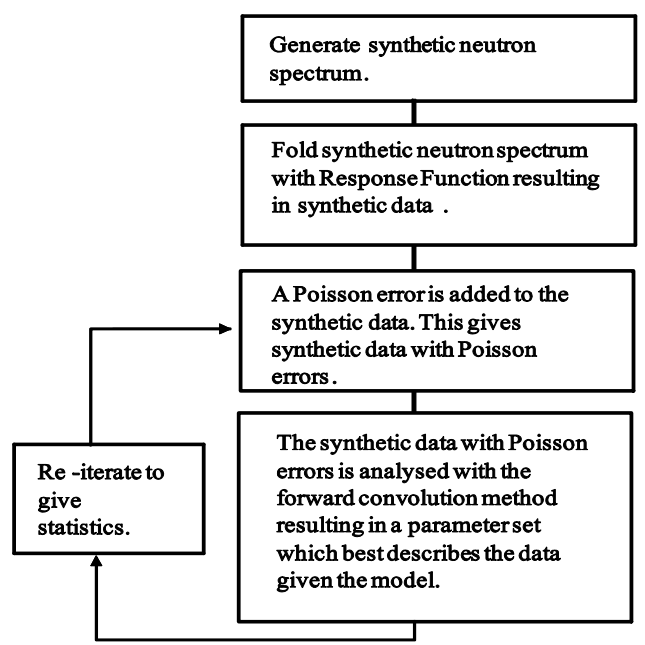

Figure 3. Overview of evaluation procedure.

\section{Neutron spectrometry techniques}

A neutron spectrometer at ITER would measure a collimated neutron flux. The neutron rate is directly proportional to $P_{\text {fusion, }}$ which implies that shorter measurement times are needed to maintain the precision when $P_{\text {fusion }}$ increases. The neutron spectrum seen by the spectrometer is line integrated along the LOS of the spectrometer through the plasma, with main contributions from high $T_{\mathrm{i}}$ (high reactivity) and high ion density regions.

In contrast to gamma spectra from tokamak plasmas, neutron spectra are generally quite "broad". Typically, an energy resolution $\left(\mathrm{FWHM} / E_{\mathrm{n}}\right)$ of about $3-5 \%$ is enough to resolve the finest features of the neutron emission spectra. An indication of the required resolving power is given by the thermal broadening which is $4 \%(\mathrm{FWHM} / \mathrm{E})$ for a DT plasma at $T_{\mathrm{i}}=9 \mathrm{keV}$. 
Five neutron-spectrometer techniques have been investigated: thin-foil magnetic proton recoil spectrometer (MPR), thin-foil proton recoil spectrometer (TPR), gamma discriminating organic scintillators (GDOS), diamonds and time-of-flight (TOF) spectrometers.

\subsection{Magnetic proton recoil technique}

In the MPR technique $[13,14]$, a collimated neutron flux impinges a thin plastic $\left(\mathrm{CH}_{2}\right)$ foil, where neutrons scatter elastically on the hydrogen nuclei of the foil. Forward scattered protons are selected by a proton collimator and allowed to enter a magnetic field, where they are spatially momentum separated. The spatial distribution of the protons is detected by a segmented detector array, a hodoscope. Due to the low efficiency, instruments utilising this technique are mainly limited by the available neutron flux. An appealing property of the MPR technique is the possibility to install interchangeable foils (with different thickness) and proton collimator diameters. The energy resolution (or efficiency) can then be chosen to optimize the instrumental performance for the measurement conditions. Furthermore, the magnetic field can be varied to shift the measured proton (neutron) energy range. The covered momentum range is superficially limited by the hodoscope size but is ultimately limited by the spectrometer's ability to give the required performance over an extended momentum (energy) range. The MPR technique has been used at JET since the late 90's [15,16,17] providing information on practically all plasma parameters accessible by neutron spectrometry.

The instrument response function (IRF) used for the MPR technique in this paper is the same as that used in the analysis of real experimental data at JET. It was obtained through measurements of the internal geometry and the magnetic field of the actual MPR spectrometer. This information was used in a Monte Carlo simulation to track protons from neutron-proton reactions in the foil to the hodoscope, as described in [13]. In the evaluation below, we use a high efficiency setting available with the present installation of the upgraded Magnetic Proton Recoil instrument (MPRu) [13] with $\varepsilon=1 \cdot 10^{-4} \mathrm{~cm}^{2}$ and resolution $\mathrm{FWHM} / E_{\mathrm{n}}=4 \%$. The monoenergetic $(14 \mathrm{MeV})$ response of the MPR is shown in Figure 4.

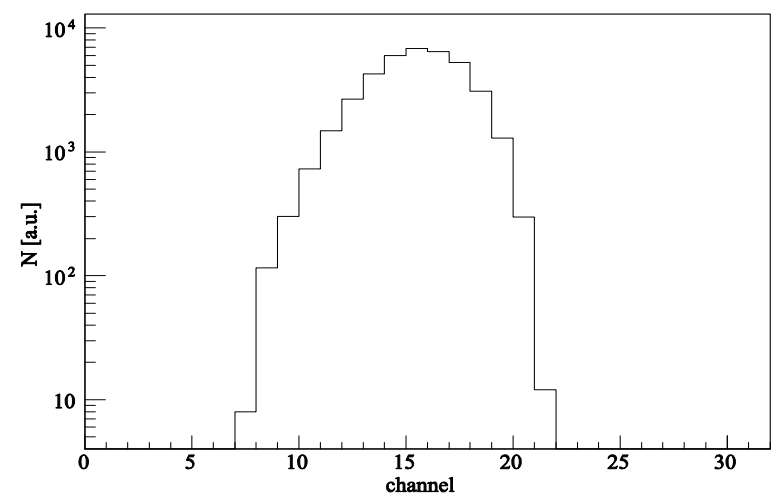

Figure 4. Instrument response of a 14-MeV neutron for the MPR technique. Note that the number of channels used here is 32 as for the present MPRu installation at JET. 


\subsection{Thin foil proton recoil technique}

In the (non-magnetic) thin-foil proton recoil, TPR, technique [18], a collimated neutron flux intersects a thin hydrogen rich foil, where elastic neutron-proton scattering takes place, as with the MPR technique. Unlike the MPR technique, no magnetic field is used. The energy measurement of the protons is instead provided by an active measurement. Recoil protons which scatter in a certain angle with respect to the incoming neutrons (or rather, the LOS) are detected by, e.g., a silicon detector. This technique is hampered by low efficiency due to the use of thin foils in the same way as the MPR. An instrument of the TPR type (TANDEM) was used during JET's main DT campaign (DTE1) in 1997 [19].

Based on the performance of $\mathrm{Si}$ detectors, measurements of mono-energetic neutrons and simulations it was concluded in [20] that the IRF of the TPR is close to Gaussian. We have followed this conclusion in this study. In [21], the optimal instrument geometry for measurement of the $\mathrm{T}_{\mathrm{i}}$ of a thermal $20 \mathrm{keV}$ DT plasma was determined in terms of foil thickness and foil-to-detector distance. This simulation was based on a commercially available annular S1 detector $\left(r_{\min }=24 \mathrm{~mm}, r_{\max }=46 \mathrm{~mm}\right.$, active area of $\left.53 \mathrm{~cm}^{2}\right)$ [22] provided by Micron Semiconductor Ltd. The simulation gave an optimal efficiency of $\varepsilon=3 \cdot 10^{-4} \mathrm{~cm}^{2}$ with an energy resolution $\left(\mathrm{FWHM} / E_{\mathrm{n}}\right)$ of $5.3 \%$ using a Gaussian shaped response. This IRF is used in this study (Figure 5).

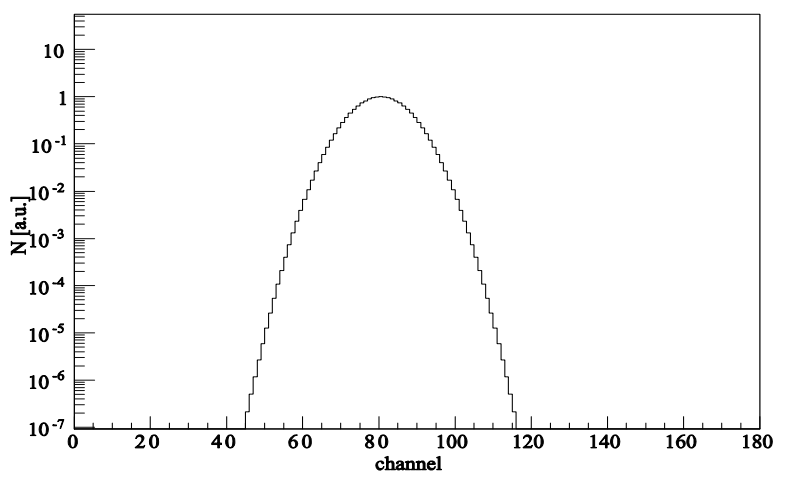

Figure 5. Instrument response of a 14-MeV neutron for the MPR technique.

\subsection{Time-of-flight technique}

Due to the continuous emission of neutrons from the plasma source, the TOF technique for fusion experiments makes use of a double-scattering approach where correlated events in two detector sets are used to measure the neutron flight times $[23,24,25,26]$. In the TOF technique discussed here, a primary and secondary detector set are aligned to a sphere, where the primary detector set is placed in the neutron beam and the secondary set is placed rotationally symmetric around the neutron beam (Figure 6a). Fast plastic scintillators are often used as detectors due to their good timing properties. The double-scattering TOF technique is inevitably associated with a disturbing background of uncorrelated, random coincidences. The background-to-signal ratio $(\mathrm{B} / \mathrm{S})$ of a TOF spectrometer due to these random events increases linearly with the neutron flux. Using the performance of the TOF spectrometer TOFOR at JET as a guide, the maximum 
count-rate capability, $C_{\max }$, of a $14-\mathrm{MeV}$ TOF is extrapolated from the DD case in [25] from $500 \mathrm{kHz}$ to $170 \mathrm{kHz}$. This decreased performance is a consequence of the restriction in count rate of the primary detectors, which is already at its maximum in TOFOR. A preserved rate in the primary detector means a three times lower rate in the secondary, due to the approximately three times lower cross section for elastic scattering of neutrons of energies $14 \mathrm{MeV}$ compared to $2.5 \mathrm{MeV}$. The TOF technique for neutron spectrometry has been used extensively at JET [23, $24,25]$ and, to a lesser degree, at JT-60U [26].

The IRF for the 14-MeV TOF technique used here is based on an MCNPX [27] simulation with a nominal flight distance of $2 \mathrm{~m}$ and scintillators coupled to photo-multiplier tubes (PMTs) as detectors. A cross section through the geometry of the MCNPX model is shown in Figure 6a. The IRF has been constructed assuming that the correlated pulse height (energy deposition) and time information is known for each scattered event in the primary and secondary detectors. This combined information allows for suppression of multi-scatter events in the detectors, as described in [28]. The response of the simulated TOF instrument to monoenergetic neutrons of $14 \mathrm{MeV}$ is shown in Figure $6 \mathrm{~b}$ with (red) and without (blue) multiscattering discrimination. The IRF employing full pulse-height discrimination (red curve in Figure 6b) is used in this study.

a)

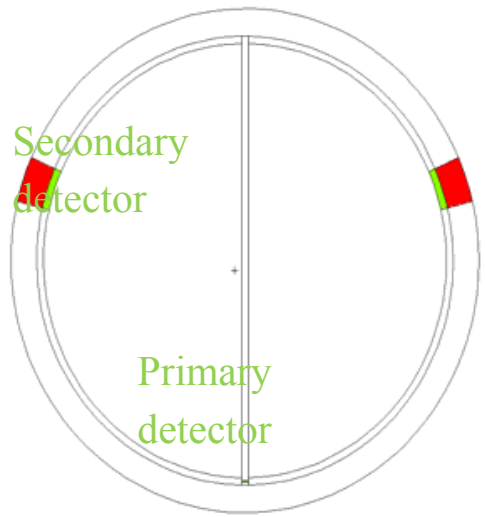

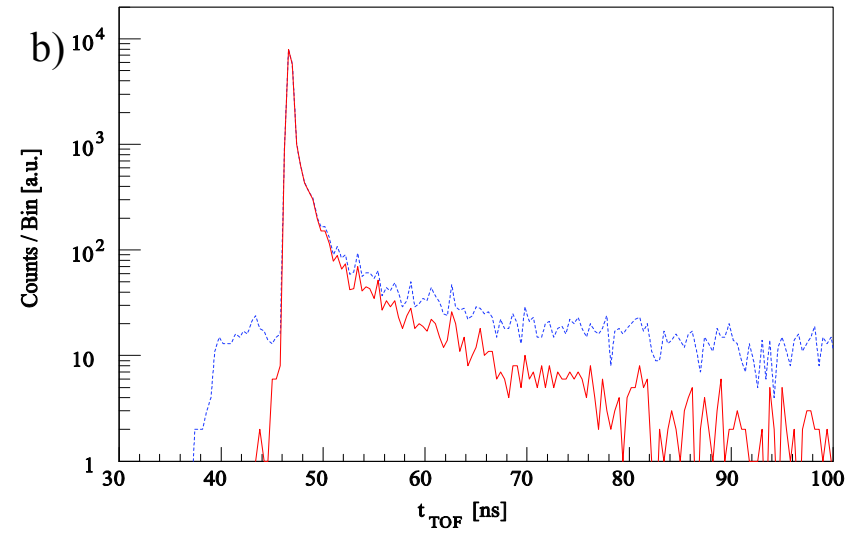

Figure 6. (a) Cross section of a MCNPX model of a TOF spectrometer with the primary and secondary detectors (green) together with the PMTs (red). The surrounding material is air (white). The black lines are geometric lines from MCNPX. (b) Instrument response of a 14-MeV neutron for the TOF technique with (red) and without (blue dashed) multi-scatter discrimination.

\subsection{Gamma discriminating organic scintillator detectors}

GDOS detectors are composed of material containing mainly hydrogen and carbon. Neutron elastic scattering on hydrogen nuclei results in recoil protons, which in turn produce scintillation light in the scintillator. In comparison to the MPR and TPR technique, there is no angular selection of the recoil protons, which results in the characteristic box-shaped IRF of the technique (Figure 7). Recoil carbon nuclei from the elastic reaction ${ }^{12} \mathrm{C}\left(\mathrm{n}, \mathrm{n}^{\prime}\right){ }^{12} \mathrm{C}$ does produce scintillating light of a much reduced intensity due to the decreased scintillation efficiency of heavier particles (quenching). However, the carbon-scattered neutron can scatter a second time on hydrogen and then deposit less energy than they would without the carbon scattering. 
Furthermore, the summed recoil proton energy increases for events with multiple small-angle scattering of a neutron on hydrogen. This effect shifts some events from lower to higher pulse heights [29]. The pulse-shapes of GDOSs detectors depend on the species of the ionizing particle. This characteristic can be used for pulse-shape discrimination to distinguish, e.g. neutrons from gammas [30]. The GDOS technique has been used for neutron spectrometry at JET [31,32] and in various nuclear and particle physics experiments, see e.g. [33].

The instrument response of GDOSs to a mono-energetic neutron emission is typically box-shaped. The IRF of a NE213 detector ${ }^{1}$, used in this study as a representative GDOS, has been characterized to a high level of detail at the Physikalisch-Technische Bundesanstalt (PTB) and the mono-energetic response of $14-\mathrm{MeV}$ neutrons is shown in Figure 7. The energy resolution is $4.1 \%$ for $14-\mathrm{MeV}$ neutrons ${ }^{2}$ [31]. The technique is limited by a maximum useful count rate capability, $C_{\max }$, of the detector for spectroscopy. In this study $C_{\max }$ of the GDOS technique is assumed to be $1 \mathrm{MHz}$ with adequate gain stability, on the basis of [34].

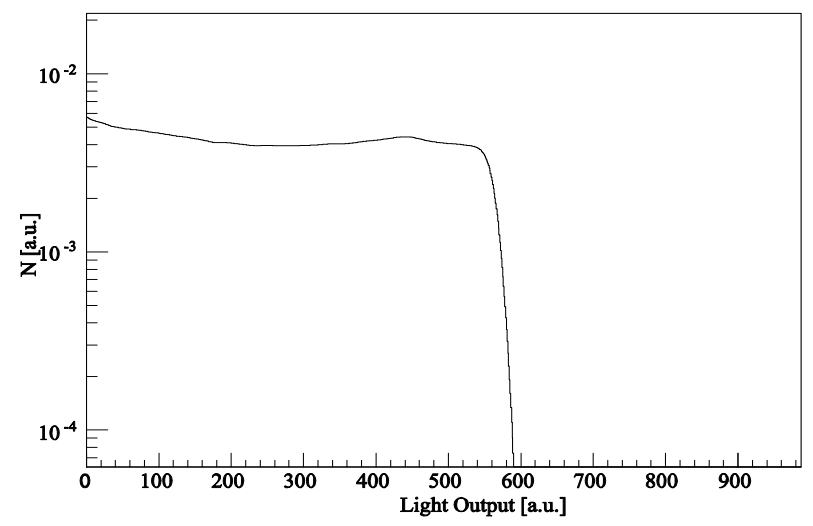

Figure 7. Instrument response of a 14-MeV neutron for the GDOS technique.

\subsection{Diamond technique}

Diamonds can be used as neutron spectrometers due to the reaction channel ${ }^{12} \mathrm{C}(\mathrm{n}, \alpha){ }^{9} \mathrm{Be}$ $\left(E_{\mathrm{th}}=6.2 \mathrm{MeV}\right)$ and their semi-conductor properties. There are, however, several competing reactions, such as ${ }^{12} \mathrm{C}\left(\mathrm{n}, \mathrm{n}^{\prime}\right){ }^{12} \mathrm{C}^{*}\left(E_{\mathrm{th}}=8.3-15.1 \mathrm{MeV}\right)$ where the excited carbon is split into three $\alpha$ particles in steps [35]. The cross sections of these two reactions are shown in Figure 8 [36]. Diamonds have been used as neutron spectrometers at JET [37], TFTR [38] and JT-60U [39].

\footnotetext{
${ }^{1}$ NE213 is a brand name GDOS, originally produced by Nuclear Enterprises, that now is commonly used as a generic name for this type of liquid scintillator.

${ }^{2}$ This resolution is obtained from the FWHM of a Gaussian fit to the "edge" of the IRF.
} 


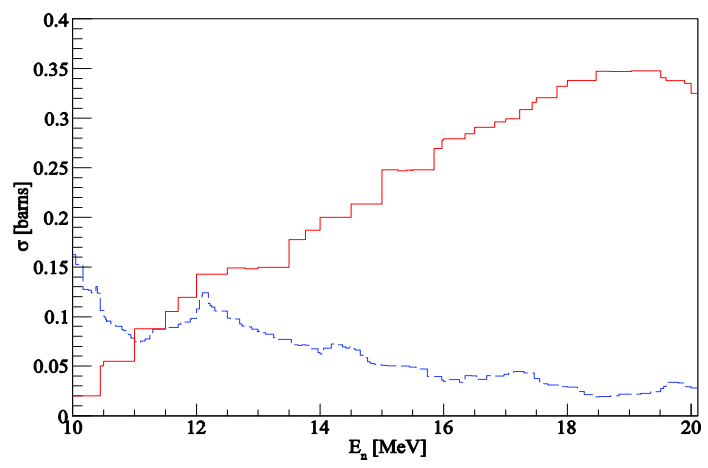

Figure 8. Cross section of ${ }^{12} \mathrm{C}(\mathrm{n}, \alpha){ }^{9} \mathrm{Be}$ (blue dashed) and ${ }^{12} \mathrm{C}\left(\mathrm{n}, \mathrm{n}^{\prime}\right) 3 \alpha$ (red) as a function of $E_{n}$.

The IRF of diamonds used in this investigation is based on measurements of quasi-mono energetic neutrons $[35,40]$. The data of [35] is used to determine the shape of the monoenergetic response for $14-\mathrm{MeV}$ neutrons. Three components are fitted to the data: two smooth step functions that decay as Gaussians, $I_{\text {step }}$ (Eq. 1), and one "double-sided Gaussian", $I_{\mathrm{dG}}$ (Eq. 2):

$$
I_{\text {step }}(x)=\left\{\begin{array}{cc}
A_{\text {step }} & \text { if } x<x_{\mathrm{m}, \text { step }} \\
A_{\text {step }} e^{-\left(\frac{\left(x-x_{\mathrm{m}, \mathrm{step}}\right)^{2}}{\left(2 \sigma_{\mathrm{step}}^{2}\right)}\right)} & \text { if } x>x_{\mathrm{m}, \mathrm{step}}
\end{array}\right.
$$

and

$$
I_{\mathrm{dG}}(x)=\left\{\begin{array}{cc}
A_{\mathrm{dG}} e^{-\left(\frac{\left(x-x_{\mathrm{m}, \mathrm{dG}}\right)^{2}}{\left(2 \sigma^{2}\right)}\right)} & \text { if } x<x_{\mathrm{m}, \mathrm{dG}} \\
A_{\mathrm{dG}} e^{-\left(\frac{\left(x-x_{\mathrm{m}, \mathrm{dG}}\right)^{2}}{\left(2 \sigma_{+}^{2}\right)}\right)} & \text { if } x>x_{\mathrm{m}, \mathrm{dG}}
\end{array}\right.
$$

where $A_{\text {step }}$ is the step-function intensity, $A_{\mathrm{dG}}$ is the intensity of the double Gaussian, $x_{\mathrm{m}, \text { step }}$ is the centre of the Gaussian decay of the smooth step function, $x_{\mathrm{m}, \mathrm{dG}}$ is the centre of the double Gaussian and, finally, $\sigma_{\text {step }}, \sigma_{-}$and $\sigma_{+}$are the standard deviations of the Gaussians in the functions. In the fit mentioned above, the intensities of the three components, the centres of the three Gaussians $\left(x_{\mathrm{m}}\right)$ and the $\sigma$ of the three components are used as free parameters. The relative intensities of the fitted components are re-normalised to fit those of [40]. The final result is shown in Figure 9, where the first step function corresponds to ${ }^{12} \mathrm{C}\left(\mathrm{n}, \mathrm{n}^{\prime}\right) 3 \alpha$, the double Gaussian to the ${ }^{12} \mathrm{C}(\mathrm{n}, \alpha){ }^{9} \mathrm{Be}$ reaction and the intermediate step function is due to non-complete charge collection. The energy dependent IRF of the diamonds is calculated by scaling the intensities of the components with their corresponding cross section and shifting the components according to the energy, resulting in the IRF matrix in Figure 9b. A small component of high-energy events above the ${ }^{12} \mathrm{C}(\mathrm{n}, \alpha){ }^{9} \mathrm{Be}$ peak is visible in the data of [40]. We ignore these events for the model of the IRF used here, assuming it is an instrumental effect, possibly pile up, that can be 
discriminated against in a future application of the technique. About $2 \%$ of all detected events originate from ${ }^{12} \mathrm{C}(\mathrm{n}, \alpha){ }^{9} \mathrm{Be}$ and we assume a $C_{\max }=1 \mathrm{MHz}[41]$.
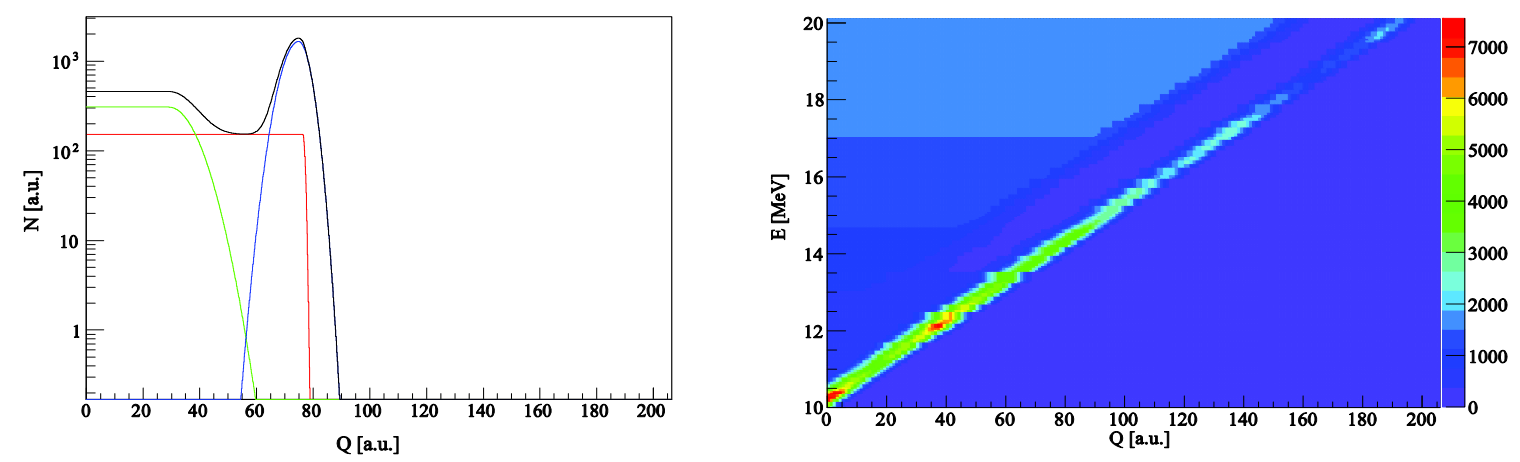

Figure 9. (a) Instrument response of a diamond to a 14-MeV neutron (black), where the two step functions (green and red) and the double Gaussian (blue) are indicated. (b) The diamond IRF efficiency matrix as a function of neutron energy and pulse height.

\section{Scenarios}

A number of reference ITER scenarios employing purely thermal as well as ICRH and NBI heating are used for various evaluation purposes, see e.g. [42]. For example, ITER reference scenario 2 is an H-mode plasma with a $Q$ (output power/input power) of 10, a plasma current, $I_{\mathrm{p}}$, of $15 \mathrm{MA}$ and a fusion power, $P_{\text {fusion, of }} 410 \mathrm{MW}$ and scenario 4 is a steady-state plasma with $Q=5, I_{\mathrm{p}}=9 \mathrm{MA}$ and $P_{\text {fusion }}=340 \mathrm{MW}$. Both scenarios have a beam heating power, $P_{\mathrm{NBI}}$, of about $35 \mathrm{MW}$ or a radio-frequency heating power, $P_{\mathrm{ICRH}}$, of $20 \mathrm{MW}[43,44]$. Here we have not attempted to model these scenarios in detail but rather used the indicated levels of power and heating as guides to the expected ITER conditions.

At ITER, a DT plasma of $500 \mathrm{MW}$, with $T_{\mathrm{i}}=20 \mathrm{keV}$, is expected to give a flux of $1 \cdot 10^{9} \mathrm{n} / \mathrm{cm}^{2} / \mathrm{s}$ (with a first wall aperture diameter of $100 \mathrm{~mm}$ ) at the dedicated HRNS position. This neutron flux is used in all scenarios described below, scaled with the reactivity as a function of $T_{\mathrm{i}}$ [45]. We have limited our study to three cases: thermal as well as ICRH and NBI heated plasmas.

\subsection{Thermal plasmas}

The neutron emission energy spectra of thermal DT plasmas can with good approximation be taken of Gaussian shape, where the width of the distribution is proportional to the square root of the temperature of the plasma, $T_{i}[46]$.

\subsection{Beam heated plasmas}

The neutron spectrum from a NBI heated DT plasma is shown in Figure 10, with $I_{\text {beam }} / I_{\text {tot }}=10 \%$ and $T_{\mathrm{i}}=20 \mathrm{keV}$, where $I$ refers to the total emission intensity (integral) of each component. In this study, a fixed shape has been used for the beam-thermal component, assuming the reference LOS of the instrument as described in Section 1, a beam injection energy of $1 \mathrm{MeV}$, an injection 
angle of the deuteron beam of $30^{\circ}$ and an electron density of $10^{20}$ [47]. The neutron emission spectrum from the beam-thermal component under these conditions has been modelled with the Monte Carlo code CONTROLROOM [48], and is shown in Figure 10.

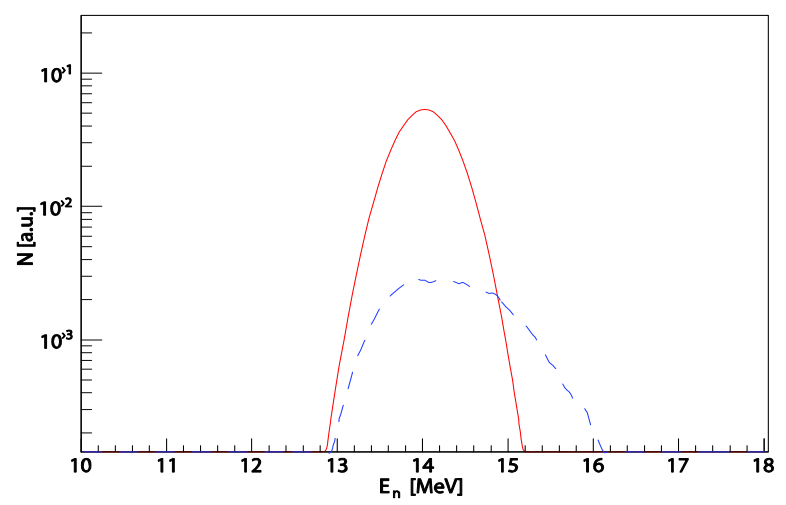

Figure 10. Neutron emission spectrum from a beam heated plasma with a thermal component (red) and a beam-thermal component (blue dashed). In this figure, $I_{\text {beam }} / I_{\text {tot }}=10 \%$ and $T_{\mathrm{i}}=20 \mathrm{keV}$.

\subsection{Ion cyclotron resonance and alpha heated plasmas}

A specific case of ICRH heating is studied here, namely the one employed in ITER standard scenario 2 (standard H-mode). The plasma parameters have been taken from simulations with the ASTRA code, see e.g. reference [49], and the tritium energy distribution has been calculated using the PION code [50]. Finally, CONTROLROOM was used to produce the neutron emission spectra.

The studied ICRH scenario has the frequency tuned to the second harmonic of the tritium ions (which coincides with the first harmonic of ${ }^{3} \mathrm{He}$ ) at $53 \mathrm{MHz}$, with a fuel mix of $n_{\mathrm{d}}=n_{\mathrm{r}} \approx n_{\mathrm{e}} / 2$ and a seeded ${ }^{3} \mathrm{He}$ population of $0-3 \%$ relative concentration. Without ${ }^{3} \mathrm{He}$, the ICRH couples to the tritium to produce pronounced tails of high energy ions and, consequently, a signature of high-energy neutrons originating from ICRH ions reacting with the thermal ion population is present in the neutron spectrum, as illustrated in Figure 11a. Introducing a small fraction of ${ }^{3} \mathrm{He}$ has the effect to reduce this high energy component as shown in Figure 11b, where $3 \%$ of the ions in the plasma are ${ }^{3} \mathrm{He}$. This can be understood by the more efficient coupling of the ICRH to the first harmonic of ${ }^{3} \mathrm{He}$ than to the second harmonic of tritium. This effect has previously been observed, see e.g. [51]. The alpha knock on component is also included in this study [15]. The alpha knock on component originates from fuel ions heated by $\alpha$ particles created in an initial $\mathrm{d}+\mathrm{t}$ reaction. This component must be considered here due to the low intensity of the ICRH component. The relative intensities of the non-thermal components of the simulation are given in Table 1. 
Table 1. Relative intensity of non-thermal spectral components in the neutron emission spectrum for different concentrations of ${ }^{3} \mathrm{He}$.

\begin{tabular}{|c|c|c|}
\hline${ }^{3} \mathbf{H e}$ & $\mathbf{I}_{\mathrm{AKN}} / \mathbf{I}_{\text {tot }}[\mathbf{\%}]$ & $\mathbf{I}_{\text {ICRH }} / \mathbf{I}_{\text {tot }}[\mathbf{\%}]$ \\
\hline $0 \%$ & 0.38 & 0.501 \\
\hline $1 \%$ & 0.38 & 0.113 \\
\hline $3 \%$ & 0.38 & 0.00984 \\
\hline
\end{tabular}
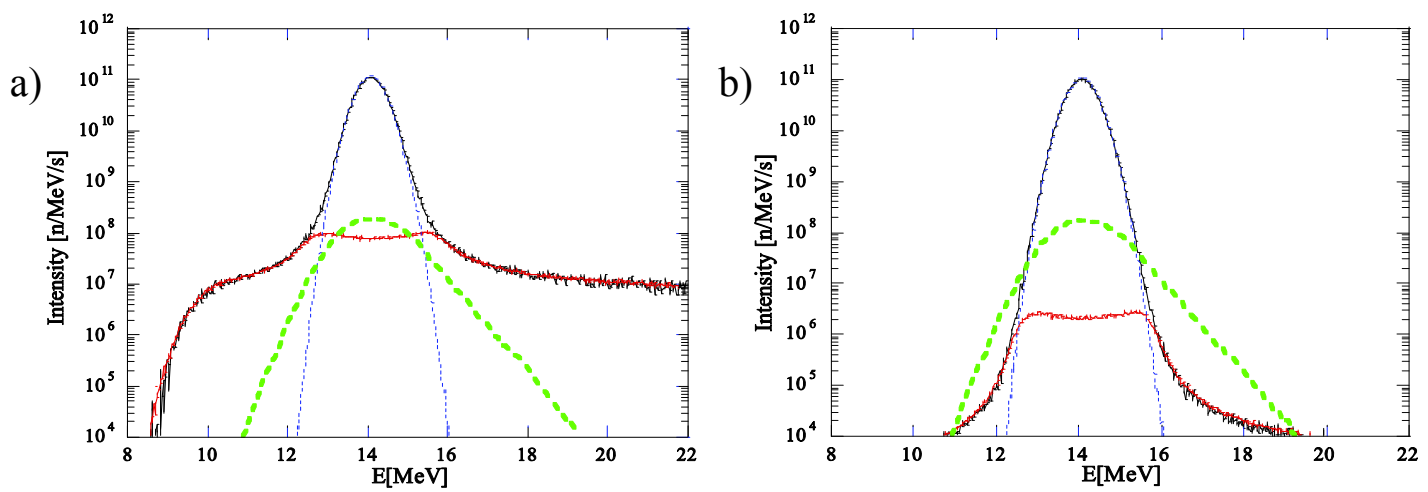

Figure 11. Neutron emission spectrum in a radial LOS from an ICRH heated plasma with a thermal component with $T_{\mathrm{i}}=\mathbf{2 0} \mathrm{keV}$ (blue), an ICRH-thermal component (red) and alpha knock on component (green) with (a) $0 \%{ }^{3} \mathrm{He}$ and (b) $3 \%{ }^{3}$ He concentration.

\section{Results}

\subsection{Thermal plasma}

In the study of pure thermal conditions, each IRF is evaluated in steps of $2 \mathrm{keV}$ over the temperature range $T_{\mathrm{i}}=1$ to $40 \mathrm{keV}$ for various integration times, $\Delta t$. The evaluation procedure was repeated 100 times per combination of $T_{\mathrm{i}}$ and $\Delta t$. The total neutron emission intensity is scaled with the reactivity with a reference emission of $1 \cdot 10^{9} \mathrm{n} / \mathrm{cm}^{2}$ at $T_{\mathrm{i}}=20 \mathrm{keV}$. The free parameters of the minimizing routine are the intensity and the temperature of the thermal component. The $2 \mathrm{D}$ precision matrix of $T_{\mathrm{i}}$ for the MPR technique is shown in Figure $12 \mathrm{a}$ as a function of $\Delta t$ and $T_{\mathrm{i}}$, i.e., $\sigma\left(\Delta t, T_{\mathrm{i}}\right)$. A precision of $10 \%$ is indicated by a red line in the figure, where the precision improves as we move from the lower left corner to the upper right. In Figure $12 \mathrm{~b}$, the time resolution curves for $10 \%$ precision measurements is shown for all the different techniques, where the maximum count rates of the GDOS, diamond and TOF techniques are set at $1 \mathrm{MHz}$ at $T_{\mathrm{i}}=40 \mathrm{keV}$. 
a)

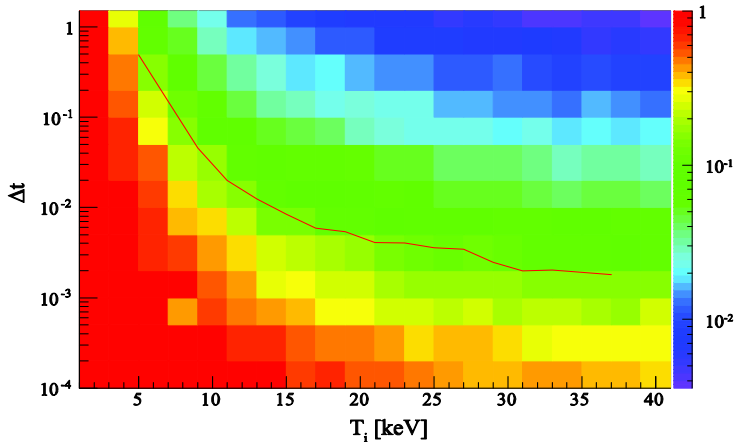

b)

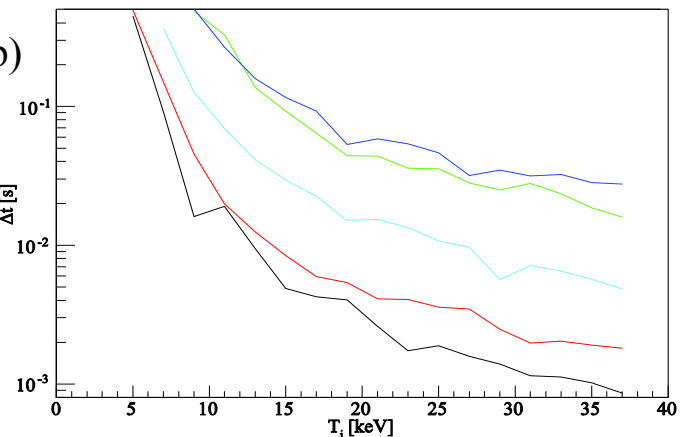

Figure 12. (a) Precision ( $1 \sigma)$ of temperature as a function of plasma temperature and time resolution for the MPR technique. The red line indicates the $10 \%$ precision region. (b) Time resolution for $10 \%$ precision as a function of temperature of a purely thermal DT plasma for TPR (black), MPR (red), TOF (turquoise), diamond (blue) and GDOSs (green).

\subsection{Neutral beam injection heated plasmas}

Two studies have been done with NBI heated plasmas: one study where the beam intensity, $I_{\text {beam, }}$ is varied relative to the intensity of the total neutron emission spectra, $I_{\text {tot }}$, and a second study where $I_{\text {beam }} / I_{\text {tot }}$ is constant and $T_{\mathrm{i}}$ is varied.

In the first NBI study, the relative beam intensity is varied in four steps $\left(I_{\text {beam }} / I_{\text {tot }}=1 \%\right.$, $5 \%, 10 \%$ and $50 \%)$ and the ion temperature is kept constant $\left(T_{\mathrm{i}}=20 \mathrm{keV}\right)$. The free parameters of the fitting routine were the intensities of the thermal-thermal and the beam-thermal components as well as $T_{\mathrm{i}}$. In Table 2 and Table 3 , the required $\Delta t$ to achieve $10 \%$ precision of $T_{\mathrm{i}}$ and $I_{\text {beam }} / I_{\text {tot }}$ is shown, respectively. Note the different behaviour of $\Delta t$ for $I_{\text {beam }} / I_{\text {tot }}$ and $T_{\mathrm{i}}$. Here, all spectrometers with a limitation in count rate reach their maximum count rate at $T_{\mathrm{i}}=20 \mathrm{keV}$.

Table 2. Required integration time to achieve $10 \%$ precision of $T_{i}$ for $I_{\text {beam }} / I_{t o t}=0 \%, 1 \%, 10 \%$ and $50 \%$. $T_{i}$ is kept constant at $20 \mathrm{keV}$.

\begin{tabular}{|l|r|r|r|r|r|}
\hline \multirow{3}{*}{$\mathbf{I R F}$} & \multicolumn{5}{|c|}{$\Delta \mathbf{t}\left(\mathbf{T}_{\mathbf{i}}\right)$} \\
\cline { 2 - 6 } & $\mathbf{I}_{\text {beam }} / \mathbf{I}_{\text {tot }}=\mathbf{0 \%}$ & $\mathbf{I}_{\text {beam }} / \mathbf{I}_{\text {tot }}=\mathbf{1 \%}$ & $\mathbf{I}_{\text {beam }} / \mathbf{I}_{\text {tot }}=\mathbf{5 \%}$ & $\mathbf{I}_{\text {beam }} / \mathbf{I}_{\text {tot }}=\mathbf{1 0} \%$ & $\mathbf{I}_{\text {beam }} / \mathbf{I}_{\text {tot }}=\mathbf{5 0 \%}$ \\
\hline MPR & $5 \mathrm{~ms}$ & $8 \mathrm{~ms}$ & $10 \mathrm{~ms}$ & $20 \mathrm{~ms}$ & $80 \mathrm{~ms}$ \\
\hline TPR & $3 \mathrm{~ms}$ & $7 \mathrm{~ms}$ & $6 \mathrm{~ms}$ & $10 \mathrm{~ms}$ & $30 \mathrm{~ms}$ \\
\hline GDOS & $50 \mathrm{~ms}$ & $100 \mathrm{~ms}$ & $100 \mathrm{~ms}$ & $200 \mathrm{~ms}$ & $1000 \mathrm{~ms}$ \\
\hline Diamond & $70 \mathrm{~ms}$ & $100 \mathrm{~ms}$ & $100 \mathrm{~ms}$ & $200 \mathrm{~ms}$ & $700 \mathrm{~ms}$ \\
\hline TOF & $10 \mathrm{~ms}$ & $6 \mathrm{~ms}$ & $30 \mathrm{~ms}$ & $40 \mathrm{~ms}$ & $100 \mathrm{~ms}$ \\
\hline
\end{tabular}


Table 3. Required integration time to achieve $10 \%$ precision of $I_{\text {beam }} / I_{\text {tot }}$ for $I_{\text {beam }} / I_{\text {tot }}=1 \%, 10 \%$ and $50 \%$. $T_{i}$ is kept constant at $20 \mathrm{keV}$.

\begin{tabular}{|l|r|r|r|r|}
\hline \multirow{3}{*}{ IRF } & \multicolumn{4}{|c|}{$\Delta \mathbf{t}\left(\mathbf{I}_{\text {beam }} / \mathbf{I}_{\text {tot }}\right)$} \\
\cline { 2 - 5 } & $\mathbf{I}_{\text {beam }} / \mathbf{I}_{\text {tot }}=\mathbf{1 \%}$ & $\mathbf{I}_{\text {beam }} / \mathbf{I}_{\text {tot }}=\mathbf{5 \%}$ & $\mathbf{I}_{\text {beam }} / \mathbf{I}_{\text {tot }}=\mathbf{1 0} \%$ & $\mathbf{I}_{\text {beam }} / \mathbf{I}_{\text {tot }}=\mathbf{5 0 \%}$ \\
\hline MPR & $4 \mathrm{~s}$ & $200 \mathrm{~ms}$ & $200 \mathrm{~ms}$ & $10 \mathrm{~ms}$ \\
\hline TPR & $2 \mathrm{~s}$ & $100 \mathrm{~ms}$ & $30 \mathrm{~ms}$ & $10 \mathrm{~ms}$ \\
\hline GDOS & $10 \mathrm{~s}$ & $1000 \mathrm{~ms}$ & $500 \mathrm{~ms}$ & $40 \mathrm{~ms}$ \\
\hline Diamond & $20 \mathrm{~s}$ & $5000 \mathrm{~ms}$ & $1000 \mathrm{~ms}$ & $200 \mathrm{~ms}$ \\
\hline TOF & $5 \mathrm{~s}$ & $500 \mathrm{~ms}$ & $200 \mathrm{~ms}$ & $30 \mathrm{~ms}$ \\
\hline
\end{tabular}

In the second NBI study, the ion temperature is varied $\left(T_{\mathrm{i}}=1-40 \mathrm{keV}, \Delta T_{i}=2 \mathrm{keV}\right)$ and the relative beam intensity is kept constant $\left(I_{\text {beam }} / I_{\text {tot }}=5 \%\right)$. In Figure $13, \Delta t$ to achieve $10 \%$ precision of $T_{\mathrm{i}}$ (panel a) and $I_{\mathrm{beam}} / I_{\text {tot }}$ (panel b) are plotted as a function of $T_{\mathrm{i}}$ for the five evaluated techniques. The free parameters of the minimizing routine were the intensity of the thermal-thermal and beam-thermal components and the temperature of the thermal component. All spectrometers with a limitation in count rate reach their maximum count rate at $T_{\mathrm{i}}=40 \mathrm{keV}$.

a)

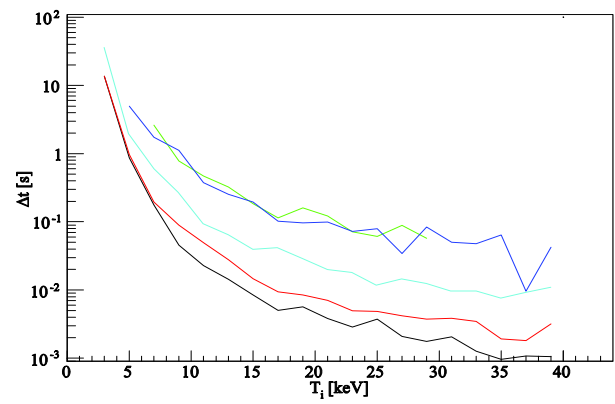

b)

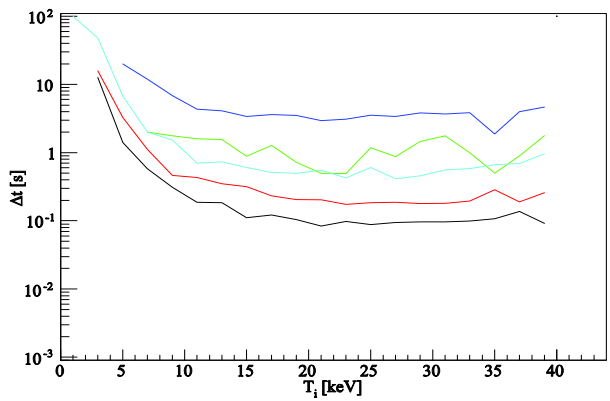

Figure 13. Integration time to achieve $10 \%$ precision of (a) $T_{i}$ and (b) $I_{\text {beam }} / I_{\text {tot }}$ as a function of temperature of a NBI heated DT plasma for TPR (black), MPR (red), TOF (turquoise), diamond (blue) and GDOS (green). $I_{\text {beam }} / I_{\text {tot }}=5 \%$ in both figures.

\subsection{Ion cyclotron resonance heated plasmas}

In the ICRH heating study, all three scenarios described in Table 1 were evaluated. In the evaluation, three parameters were used as free variables: $T_{\mathrm{i}}$ as well as the intensities of the thermal-thermal and the thermal-ICRH, $I_{\mathrm{ICRH}}$, component. The intensity of the AKN component was fixed relative to that of the thermal-thermal component. None of the techniques could determine $I_{\text {ICRH }}$ in the presence of $3 \%{ }^{3} \mathrm{He}$. 
Table 4. Required integration time to achieve $10 \%$ precision of $I_{\text {ICRH }} / I_{\text {tot }}$ for concentrations of $0 \%$ and $1 \%$ of ${ }^{3} \mathrm{He}$.

\begin{tabular}{|l|r|r|}
\hline \multicolumn{1}{|c|}{ IRF } & $\begin{array}{c}\Delta t\left(\mathbf{I}_{\mathbf{I C R H}} / \mathbf{I}_{\text {tot }}\right) \\
\mathbf{0 \%}{ }^{\mathbf{3}} \mathbf{H e}\end{array}$ & $\begin{array}{c}\Delta \mathrm{t}\left(\mathbf{I}_{\mathbf{I C R H}} / \mathbf{I}_{\text {tot }}\right) \\
\mathbf{1 \%}{ }^{{ }^{3}} \mathbf{H e}\end{array}$ \\
\hline MPR & $0.9 \mathrm{~s}$ & $9 \mathrm{~s}$ \\
\hline TPR & $0.3 \mathrm{~s}$ & $2 \mathrm{~s}$ \\
\hline GDOS & $0.8 \mathrm{~s}$ & $50 \mathrm{~s}$ \\
\hline Diamond & $4 \mathrm{~s}$ & $10 \mathrm{~s}$ \\
\hline TOF & $1 \mathrm{~s}$ & \\
\hline
\end{tabular}

\section{Discussion}

For the three plasma scenarios studied here the thin-foil techniques perform best closely followed by the TOF technique, in terms of $\Delta t$. In the thermal scenario $\Delta t$ decreases with $T_{\mathrm{i}}$, which is an expected effect since the reactivity decreases with lower $T_{\mathrm{i}}$ (which reduces the neutron flux at the detector) and the limited resolution of the techniques put higher demands on good statistics in order to accurately resolve the width of the narrow Gaussian peak. In this study we conclude that for purely thermal plasmas, the thin-foil techniques can meet the ITER requirement ( $10 \%$ precision of $T_{\mathrm{i}}$ with time resolution of $\left.100 \mathrm{~ms}\right)$ when $T_{\mathrm{i}}>7 \mathrm{keV}$. For the TOF technique, $T_{\mathrm{i}}$ must be higher than $11 \mathrm{keV}$, and for the diamond and GDOSs, $T_{\mathrm{i}}>15 \mathrm{keV}$, to meet the ITER requirements.

In the NBI heating scenario, the case of varied relative intensity of the beam component and fixed temperature, one observes that the $\Delta t$ of $T_{\mathrm{i}}$ increases with increased $I_{\text {beam }} / I_{\text {tot }}$ as expected (see Table 2). The TPR, MPR and TOF techniques meet the ITER requirements for all levels of $\mathrm{I}_{\text {beam, }}$, whereas the GDOS and diamond techniques only reach the ITER requirement for $I_{\text {beam }} / I_{\text {tot }}<5 \%$. In Table 3, it should be noted that none of the techniques can measure $I_{\text {beam }} / I_{\text {tot }}=1 \%$ with a time resolution less than a second. However, with $I_{\text {beam }} / I_{\text {tot }}=5 \%$, the TPR technique achieves the ITER requirements and with $I_{\text {beam }} / I_{\text {tot }}=50 \%$ the TPR, MPR, GDOS and the TOF techniques all meet the ITER requirements.

In the second beam heating study, where $T_{\mathrm{i}}$ is varied and $I_{\text {beam }} / I_{\text {tot }}=5 \%$, it is shown that only the TPR technique meets the ITER requirement for $T_{\mathrm{i}}>15 \mathrm{keV}$. It is interesting to note that when determining $T_{\mathrm{i}}$, the diamond and the GDOS techniques achieve the same time resolution. However, to resolve $I_{\text {beam }} / I_{\text {tot }}$ the required integration time is more than twice as long for the diamond compared to the GDOS. This can be understood in terms of the decreased cross section of the ${ }^{12} \mathrm{C}(\mathrm{n}, \alpha){ }^{9} \mathrm{Be}$ reaction at higher neutron energies.

In the ICRH study, none of the techniques achieve the ITER requirements. It should be noted that in the ${ }^{3} \mathrm{He}$ free ICRH plasmas, the GDOS technique has a better time resolution than the MPR technique. This can be understood from the restricted range of detected proton recoil energies of the present implementation of the MPRu hodoscope. 
The IRFs used in this study are of different levels of maturity, something that should be considered when interpreting the results. The IRFs of the MPR and GDOS techniques have been measured and experimentally verified; in fact the IRFs used for these two techniques are basically the same as used in the analysis of real experimental data. In comparison, the IRFs of the other three techniques are on a much less mature level, and several simplifications have been introduced in their descriptions.

The IRF of the TPR technique is represented by an ideal Gaussian function. There is some experimental evidence for this assumption [20] although the presence of low-energy tails, as seen in for example the diamond IRF, could worsen the performance somewhat. To obtain a more realistic IRF, the technique should be examined more thoroughly in terms of detector response as a function of neutron energy, background sensitivity and effects of surrounding material such as radiation shielding. However, the present study clearly indicates that the TPR technique is a promising candidate for a HRNS, which could possibly perform on par with the more established MPR technique. In addition, its non-magnetic character and smaller size and weight would considerably reduce the interfacing issues at ITER compared to the MPR.

The diamond IRF used here was modelled from experimental data from two different (mono-energetic) measurements. Clearly, measurements covering a wider range of neutron energies would give a more complete picture of the IRF and could possibly alter the result of this study. Due to the intrinsically very fast signals of diamond detectors, the count rate limit of $1 \mathrm{MHz}$ used here could be on the pessimistic side, if adequate signal processing electronics can be developed. In addition, the small size of diamond detectors could allow for arrays of such devices to be used in parallel, thereby increasing the total sustainable rate of the combined system even further. It is also possible that further advances in the manufacturing of artificial, carbon vapour deposit diamonds will produce samples of consistently better quality than seen so far. This could further enhance the performance of this technique. Finally, one should also note the advantages that the diamond technique offers in terms of radiation hardness and robustness, qualities that could be particularly important when equipping the ITER neutron camera with suitable detectors.

The TOF is a well established technique for neutron spectrometry, in particular concerning measurements of $2.5 \mathrm{-MeV}$ neutrons from D plasmas. In this study, we have assumed that suitable signal processing electronics can be employed and therefore used both the pulse-height and the time information of correlated scattering events in the two detector sets to discard unphysical events. This allows us to resolve components of low intensity relative to the dominant thermal-thermal component, without the interference of random coincidences which is otherwise a limiting factor for this technique. However, it should be pointed out that such signal processing capabilities have not been experimentally tested on any existing fusion TOF system and further studies must be made in order to verify the feasibility of this technique and its detailed performance. 


\section{Conclusions}

The performance of five different neutron-spectrometric techniques has been examined under realistic ITER conditions: the gamma discriminating organic scintillator, the diamond, the timeof-flight and two thin-foil techniques. Three scenarios have been simulated: thermal as well as NBI and ICRH heated plasma conditions. A first wall aperture of $50 \mathrm{~mm}$ radius was used. In terms of time resolution, the two thin-foil techniques, MPR and TPR, show the best performance in (almost) all evaluated plasma scenarios. Based on the present study, a safe choice for a 14-MeV HRNS on ITER would therefore be a thin-foil proton recoil spectrometer of the MPR type, provided that interfacing issues can be solved. Even if it is a much less mature technique, the good performance indicated here and the much reduced interfacing issues for the TPR system should motivate further simulation and experimental studies of this technique. Finally, due to the quite different levels of maturity of the response functions of the different techniques, further technical developments and experimental experience could change the situation for some of the techniques, in particular for diamonds.

\section{Acknowledgements}

This work, supported by the European Communities under the contract of Association between EURATOM and VR, was carried out within the framework of the European Fusion Development Agreement. The views and opinions expressed herein do not necessarily reflect those of the European Commission.

\section{References}

[1] H. Sjöstrand et al., Fusion Sci. and Tech. 57 (2010) 162.

[2] M. Tardocchi et al., Rev. Sci. Instrum. 75 (2004) 661.

[3] H. Henriksson et al., Nucl. Fusion, 46 (2006) 244.

[4] J. Källne et al., Rev. Sci. Instrum., 62 (1991) 2871.

[5] C. Hellesen et al., Nucl. Fusion, 50 (2010) 1.

[6] M. Gatu Johnson et al., Nucl. Fusion, "Neutron emission from beryllium reactions in JET deuterium plasmas with ${ }^{3} \mathrm{He}$ minority" In press (2010)

[7] A.J.H. Donné et al., Nucl. Fusion 47 (2007) S337.

[8] G. Ericsson et al., EFDA-JET-CP(07)04/08

[9] T.Craciunescu et al., Nucl. Instrum. Meth. A, 605 (2009) 374.

[10] E. Andersson Sundén et al., JET Pre-print EFDA-JET-CP(07)04/10

[11] E. Ronchi et al., JET Pre-print EFDA-JET-CP(08)06-01

[12] W. Cash, Astrophys. Journal, 228 (1979) 939.

[13] E. Andersson Sundén et al., Nucl. Instr. And Meth. A, 610 (2009) 682.

[14] J. Frenje et al., Rev. Sci. Instrum., 79 (2008) 10 E502.

[15] J. Källne et al., Phys. Rev. Lett., 85 (2000) 1246.

[16] M. Tardocchi et al., Rev. Sci. Instrum., 72 (2001) 836. 
[17] H. Henriksson et al., Rev. Sci. Instrum., 72 (2001) 832.

[18] N.P. Hawkes et al., Rev. Sci. Instrum., Vol. 70 (1999) 1134.

[19] N.P. Hawkes et al., Nucl. Instrum. and Meth. A, 476 (2002) 490.

[20] N.P. Hawkes et al., Nucl. Instrum. and Meth. A, 476 (2002) 506.

[21] S. Conroy et al., Rev. Sci. Instrum., 79 (2008) 10E508.

[22] http://www.micronsemiconductor.co.uk/pdf/s.pdf

[23] T. Elevant, Nucl. Instrum. and Meth. A, 476 (2002) 485.

[24] O.N. Jarvis, Nucl. Instrum. and Meth. A, 476 (2002) 474.

[25] M. Gatu Johnson et al., Nucl. Instrum. Meth. A, 591 (2008) 417.

[26] Y.Shibata et al., Rev. Sci. Instrum., 72 (2001) 828.

[27] MCNPX User's Manual, Version 2.5.0, LA-CP-05-0369, edited by D. B.

Pelowitz, (2005).

[28] E. Andersson Sundén et al., Nucl. Instrum. Meth. A, "Instrumentation for neutron emission spectrometry in use at JET" (2010)

[29] S. Neumann et al., Nucl. Instrum. Meth. A., 476 (2002) 353.

[30] B. Esposito et al., Nucl. Instrum. Meth. A., 518 (2004) 626.

[31] Yu.A.Kaschuck et al, 31st EPS Conference on Plasma Phys. London (2004) P-5.174.

[32] A. Zimbal et al., Rev. Sci. Instrum. 75 (2004) 3553.

[33] F.D. Brooks et al., Radiat. Prot. Dosimetry, 126 (2007) 218.

[34] D. Marocco et al., IEEE Nucl. Sci., 56 (2009)

[35] M. Pillon et al., Nucl. Instrum. and Meth. B, 101 (1995) 473.

[36] ENDF7 (http://www.nndc.bnl.gov/exfor/endf00.jsp)

[37] A.V. Krasilnikov et al., Nucl. Instrum. And Meth. A, 476 (2002) 500.

[38] A.V. Krasilnikov et al., Rev. Sci. Instrum., 68 (1997) 553.

[39] M. Isobe et al., Fusion Engi. and Des., 34-35 (1997) 573.

[40] G.J. Schmid et al., Nucl. Instrum. and Meth. A, 527 (2004) 554.

[41] A.V. Krasilnikov et al., Rev. Sci. Instrum., 68 (1997) 1720.

[42] V. Parail et al., Nucl. Fusion, 49 (2009) 075030

[43] M. Shimada et al., Nucl. Fusion, 47 (2007) S1.

[44] C. Gormezano et al., Nucl. Fusion, 47 (2007) S285.

[45] H.S. Bosch et al., Nucl. Fusion, 32 (1992) 611.

[46] H.Brysk, Plasma Phys., 15 (1973) 611.

[47] G. Gorini et al., EFDA-JET-CP(06)03-13

[48] L. Ballabio et al., Nucl. Fusion 39 (1998) 1723.

[49] Polevoi et al., J. Plasma Fusion Res., 5 (2002) 82.

[50] L.-G. Eriksson et al., Nucl. Fusion 33 (1993) 1037.

[51] D.F.H. Start et al., Nuclear Fusion, 39 (1999) 321. 